\title{
Instant Messaging: an Innovative Way to Recruit Primary Care Providers for Qualitative Research
}

\author{
Justina Wu, MPH ${ }^{7}$, Eleanor T. Lewis, PhD ${ }^{7,2}$, Paul G. Barnett, $P h D^{1,3}$, and \\ Andrea L. Nevedal, $P h D^{7}$
}

${ }^{1}$ Center for Innovation to Implementation, VA Palo Alto Healthcare System, Menlo Park, CA, USA; ${ }^{2}$ Program Evaluation and Resource Center, VA Office of Mental Health and Suicide Prevention, Menlo Park, CA, USA; ${ }^{3}$ Health Economics Resource Center, VA Palo Alto Healthcare System, Menlo Park, CA, USA.

J Gen Intern Med 35(5):1612-4

DOI: $10.1007 / \mathrm{s} 11606-019-05533-2$

(c) Society of General Internal Medicine (This is a U.S. government work and not under copyright protection in the U.S.; foreign copyright protection may apply) 2019

\section{INTRODUCTION}

Recruiting primary care providers (PCPs) for participation in health services research is a major challenge. PCPs report barriers such as time constraints ${ }^{1-3}$, low interest or buy-in for the research topic ${ }^{3}$, and inadequate rapport with researchers ${ }^{2}$. Busy PCPs are overloaded with alerts and reminders ${ }^{4}$, and hard to reach via phone ${ }^{5}$ or email, resulting in asynchronous communication and lost, forgotten, or ignored messages. In integrated health care systems such as the Veterans Health Administration (VA), organization-wide instant messaging (IM) allows real-time communication between employees. We describe a novel use of IM to increase PCP participation in interviews for health services research.

\section{METHODS}

As part of a mixed-methods study, we emailed eligible VA PCPs between June 2017 and September 2018 inviting them to participate in a semi-structured telephone interview about their use of lumbar spine magnetic resonance imaging for managing uncomplicated, acute low-back pain. Initially, researchers emailed follow-up messages to PCPs who did not respond. After 5 months of poor response rates using emailonly recruitment, we began using IM (Skype for Business, Microsoft Corp, 2018) to follow-up with PCPs. The Stanford Institutional Review Board approved this study modification (no. 38033) ${ }^{6}$.

Two researchers spent about $10 \mathrm{~h}$ per week recruiting PCPs through IM (Fig. 1). Using IM software, we searched for PCPs' names then sent a brief message to PCPs whose status was "available" to re-introduce the study and request

Received July 26, 2019

Revised October 4, 2019

Accepted October 29, 2019

Published online November 25, 2019 participation. If the PCP agreed, we asked for their availability for a 15- to 30-min telephone interview. We sent up to three IMs to "available" PCPs who did not respond to our IM, before considering them a passive decline. PCPs whose status was "unavailable" were noted, and we checked again another time.

\section{RESULTS}

We sent invitation emails to 452 PCPs. Of these, 55 completed interviews and 66 declined. During the first 5 months of the study when we used email-only recruitment, only 3 PCPs were interviewed and 5 declined. In months 6 through 16, we used IM follow-up with 357 PCPs who did not respond to the initial email, and 52 PCPs were interviewed and 61 declined (Fig. 1). During the 11 months of IM follow-up, we conducted an average of 5 interviews per month, including 1 month when we completed 15 interviews (Fig. 2).

Using organization-wide IM increased interview participation, defined as percent of sample interviewed, from $3.2 \%$ using email-only recruitment to $14.6 \%$ using IM follow-up. Instant messaging also improved the number of responses of any kind from $8.4 \%$ using email-only to $38.4 \%$ using IM.

Reasons for declining, offered via IM, included "overwhelmed with current workload," "the local facility will [not] allow me time to participate", or "not really interested." Some PCPs indicated they were interested but busy: "Seeing patients, check back tomorrow" or "I will get back to you," and sometimes offered times when they could be reached. Other PCPs replied that they were free but would not be available later, so we interviewed them immediately.

\section{DISCUSSION}

Email-only recruitment resulted in low response rates, and PCPs were more responsive to IM. IM is more noticeable on-screen than email and encourages greater immediacy of response. PCPs can respond briefly over IM ${ }^{7}$, ignore the message, or respond later, but they may feel more compelled to answer knowing someone is waiting for a response. IM allowed PCPs to participate immediately, rather than 


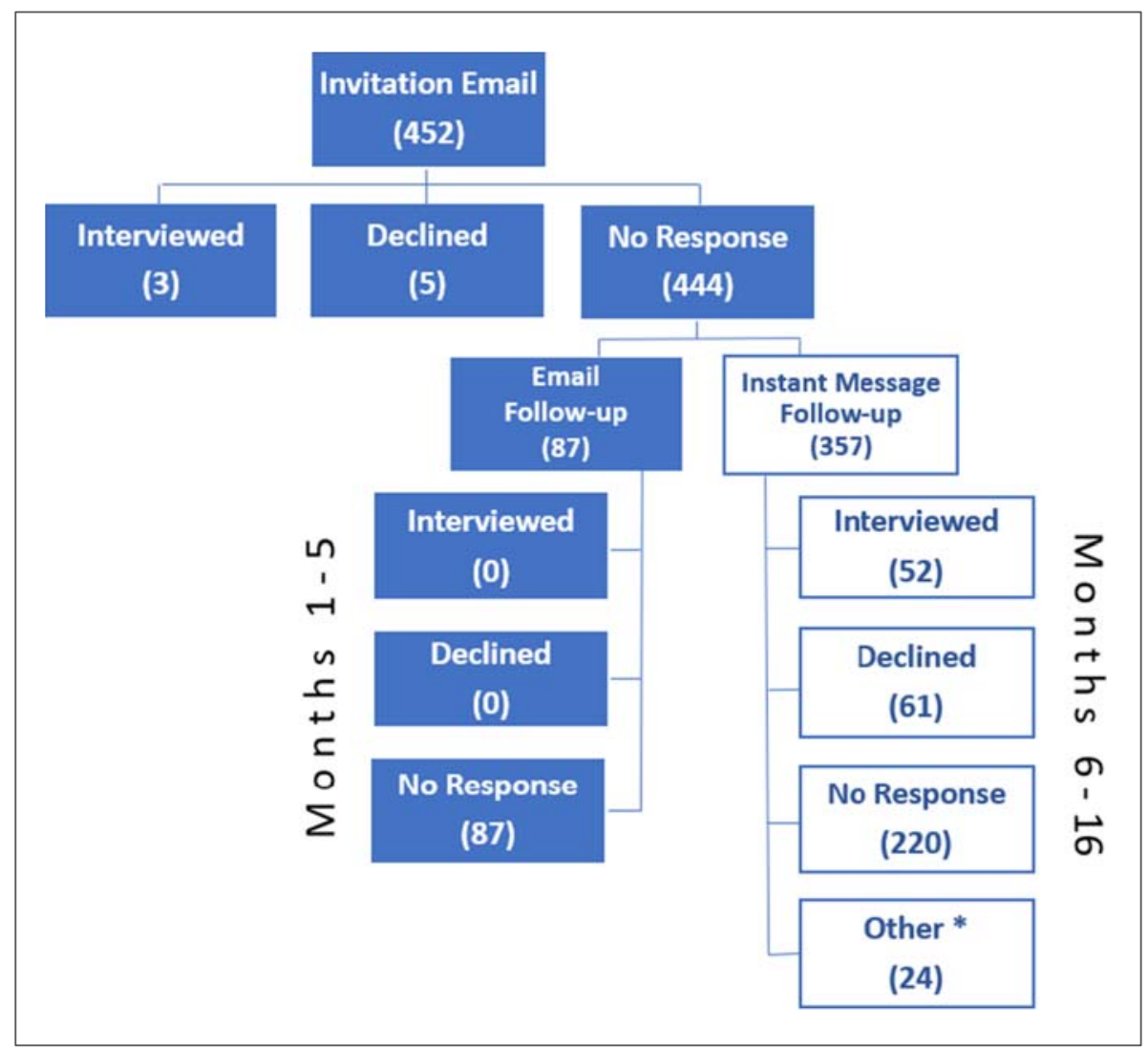

*Interested but incomplete or unscheduled interviews

Figure 1 Comparison of email versus instant message follow-up strategies. The asterisk symbol indicates interested but incomplete or unscheduled interviews

postponing for a future date. The casual nature of IM also helped build rapport, as some PCPs engaged in friendly banter with the researcher.

IM follow-up recruitment required more active participation from researchers but was more efficient than email. Sending an IM when someone was online yielded more responses, drastically improved interview participation rates, and reduced the overall time spent recruiting.

Although other research describes IM use in clinical practice or how electronic platforms are used for recruitment, this

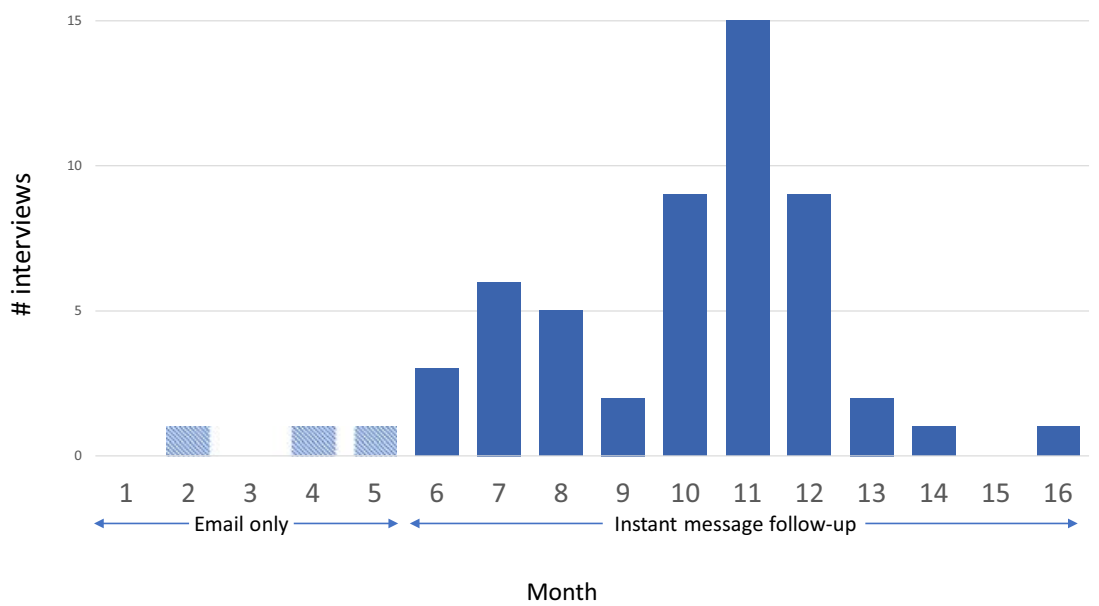

Figure 2 Interviews per month across recruitment period and strategy 
is the first study we know of using IM to recruit PCPs. Our study provides evidence that PCPs are receptive to recruitment via IM; however, additional research is needed to assess the acceptability of IM to providers. In addition, researchers will need approval from Human Subjects Research Committees to ensure IM is used judiciously. Organization-wide IM recruitment shows promise for increasing PCP participation rates in health services research.

Acknowledgments: We are grateful to the primary care providers who participated in this study and shared their frontline experiences with us. We acknowledge Josephine Jacobs, Jeanie Lo, Samantha Illarmo, Roger Chou, Jeffrey Jarvik, Melanie Jeske, Derek Boothroyd, , Lucile Burgo, Ajo John, Friedhelm Sandbrink, Kate Stanitski, and Suzanne Tharin for their important contributions to the larger study.

Corresponding Author: Justina Wu, MPH; Center for Innovation to Implementation, VA Palo Alto Healthcare System, Menlo Park, CA, USA (e-mail: Justina.Wu@va.gov).

Funding Information This work was financially supported by an Investigator Initiated Research grant no. 15-450 from the Department of Veterans Affairs Health Services Research and Development Program.

\section{Compliance with Ethical Standards:}

The Stanford Institutional Review Board approved this study modification (no. 38033).

Disclaimer: The opinions expressed in this article are those of the authors and do not reflect the official position of the US Department of Veterans Affairs.
Conflict of Interest: The authors declare that they do not have a conflict of interest.

\section{REFERENCES}

1. Asch S, Connor SE, Hamilton EG, Fox SA. Problems in recruiting community-based physicians for health services research. J Gen Intern Med. 2000;15(8):591-599. doi:https://doi.org/10.1046/j.1525-1497. 2000.02329.x

2. Johnston S, Liddy C, Hogg W, Donskov M, Russell G, Gyorfi-Dyke E. Barriers and facilitators to recruitment of physicians and practices for primary care health services research at one centre. BMC Med Res Methodol. 2010;10(1):109. doi:https://doi.org/10.1186/1471-2288-10109

3. Sahin D, Yaffe MJ, Sussman T, McCusker J. A mixed studies literature review of family physicians' participation in research. Fam Med. 2014;46(7):503-514.

4. Backman R, Bayliss S, Moore D, Litchfield I. Clinical reminder alert fatigue in healthcare: a systematic literature review protocol using qualitative evidence. Syst Rev. 2017;6(1):255. doi:https://doi.org/10.1186/s13643017-0627-z

5. Hassol A, Walker JM, Kidder D, et al. Patient experiences and attitudes about access to a patient electronic health care record and linked web messaging. J Am Med Inform Assoc. 2004;11(6):505-513. doi:https://doi. org/10.1197/jamia.M1593

6. Nevedal AL, Lewis ET, Wu J, et al. Factors influencing primary care providers' unneeded lumbar spine MRI orders for acute, uncomplicated low-back pain: a qualitative study. $J$ Gen Intern Med. (accepted).

7. Cameron AF, Webster J. Unintended consequences of emerging communication technologies: instant Messaging in the workplace. Comput Hum Behav. 2005;21(1):85-103. doi:https://doi.org/10.1016/j.chb.2003.12. 001

Publisher's Note Springer Nature remains neutral with regard to jurisdictional claims in published maps and institutional affiliations. 